\title{
Digestibilité comparée de quatre céréales (avoine, orge, maïs, blé), selon le mode de présentation, chez le poney
}

\author{
R. WOLTER *, J.P. VALETTE *, Andrée DURIX**, \\ J.C. LETOURNEAU ** et Monique CARCELEN $* *$ \\ avec la collaboration de A. ViLLARD ** et A. BRUNY ** \\ * I.N.R.A., Laboratoire de Nutrition et Alimentation \\ Ecole nationale vétérinaire d'Alfort \\ 7, avenue du Général-de-Gaulle, F 94704 Maisons-Alfort Cedex \\ ** I.N.R.A., Laboratoire de Nutrition et Alimentation \\ Ecole nationale vétérinaire de Lyon \\ Route de Saint-Bel, Marcy-l'Etoile, F 69260 Charbonnières-les-Bains
}

\section{Résumé}

Six poneys placés en cages à métabolisme reçoivent à raison de $2 \mathrm{~kg} / \mathrm{animal} / \mathrm{jour}$, en deux repas, l'une des quatre céréales suivantes : avoine, orge, maïs et blé, présentées entières, aplaties, concassées ou trempées, en égalisant le taux de cellulose des régimes grâce à une complémentation ajustée en paille.

Les résultats font ressortir que :

- Le conditionnement a peu d'influence sur la digestibilité d'une céréale donnée.

- L'avoine est moins bien digérée que les autres céréales, notamment en ce qui concerne la matière sèche et la matière organique, ce qui confirme sa moindre valeur énergétique.

Par ailleurs, le contrôle de l'évolution des taux sanguins d'acide lactique et de glucose ne permet de déceler aucune différence en fonction de la céréale consommée.

\section{Introduction}

Pour les équidés, les tables de digestibilité établies chez les ruminants conviennent mal, d'autant plus que le taux alimentaire de cellulose est plus élevé, compte tenu de la moindre aptitude à digérer celle-ci (Hintz, 1969 ; VANDER NooT \& Gilbraith, 1970 ; VANDER NoOT \& Trout, 1971).

De ce fait, des études spécifiques de la digestibilité des aliments cellulosiques doivent être conduites chez le cheval, ou chez le poney qui en est un bon modèle expérimental (Slade \& Hintz, 1969 ; Wolter \& ChaAbouni, 1979). C'est ainsi que 
furent bien appréciées les graminées et les légumineuses prairiales (FonNESBECK et al., 1967 ; Darlington \& Hershberger, 1968 ; FonNESBECK, 1968 et 1969 ; VANDER Noot \& Gilbraith, 1970), les fourrages broyés et/ou granulés (Hintz \& Loy, 1966; Haenlein, Holdren \& Yoon, 1966 ; Wolter, Durix \& Letourneau, 1974 et 1975), le maïs fourrage (Schurg et al., 1977), la paille, le son de blé, les pulpes de betteraves ou de raisin, les pellicules de soja, le marc de pomme, le tourteau de pépins de raisin... (Wolter et al., 1979 et 1980).

En ce qui concerne les grains, qui ont la réputation d'être bien digérés à condition de broyer les grains durs, voire d'aplatir l'avoine quand la mastication est insuffisamment efficace, quelques essais ont déjà été conduits pour juger de l'influence de la présentation de l'avoine sur sa digestibilité chez le poney (CENNI et al., 1973; Bouwman, 1978). Dans le présent travail, nous comparons l'avoine, l'orge, le maïs et le blé, sous diverses formes (entière, aplatie, trempée ou concassée), dans des régimes égalisés quant au taux de cellulose afin de prévenir le déclenchement des troubles digestifs.

\section{Conditions expérimentales}

\section{A. Animaux et aliments}

Six poneys mâles, pesant $180 \mathrm{~kg}$ en moyenne, sont placés en cages à métabolisme et reçoivent successivement chacun des 4 régimes à base de céréales et de paille de blé à raison de $40 \mathrm{~g} / \mathrm{kg} \mathrm{PV}^{0,75} /$ jour, soit $2000 \mathrm{~g} / \mathrm{animal} / \mathrm{jour}$, répartis en 2 repas, pendant une période d'adaptation de 3 semaines, suivie d'une période de récolte des matières fécales de 6 jours.

Les céréales et la paille, dont les compositions chimiques sont rapportées aux tableaux 1 a et $1 \mathrm{~b}$, sont fournies séparément.

Les tableaux 2 a et 2 b récapitulent la nature des régimes et leur composition analytique calculée.

\section{B. Méthodes d'analyses}

Les digestibilités sont déterminées par la méthode de récolte totale des fèces. Les aliquotes des prélèvements quotidiens sont séchés à l'étuve à $80^{\circ} \mathrm{C}$ pendant 48 heures, homogénéisés et soumis aux analyses suivantes :

- protéines totales $(\mathrm{N} \times 6,25)$, par la méthode de Kjeldahl;

- cellulose brute, par la méthode de Weende;

- constituants pariétaux, selon la méthode de Van Soest (1963);

- matières minérales totales, après incinération à $550^{\circ} \mathrm{C}$ pendant 5 heures ;

- calcium, par photométrie d'émission de flamme ;

- phosphore, par colorimétrie à $430 \mathrm{~nm}$ avec le réactif vanadomolybdique ;

- amidon, après hydrolyse enzymatique à l’amyloglucosidase et dosage du glucose. 


\section{TABLEAU la}

Composition analytique générale des céréales et de la paille (p. 100).

Analytical composition of cereals and straw.

\begin{tabular}{|c|c|c|c|c|c|}
\hline & $\begin{array}{c}\text { Avoine } \\
\text { Oat }\end{array}$ & $\begin{array}{c}\text { Orge } \\
\text { Barley }\end{array}$ & $\begin{array}{l}\text { Maïs } \\
\text { Maize }\end{array}$ & $\begin{array}{c}\text { Blé } \\
\text { Wheat }\end{array}$ & $\begin{array}{l}\text { Paille } \\
\text { de blé } \\
\text { Wheat } \\
\text { straw }\end{array}$ \\
\hline $\begin{array}{l}\text { Matière sèche } \ldots \ldots \ldots \ldots \\
\text { Dry matter }\end{array}$ & 89,3 & 91,8 & 87,7 & 87,6 & 93,9 \\
\hline \multicolumn{6}{|l|}{ p. 100 de M.S. } \\
\hline Amidon - Starch .... & 35,2 & 54,6 & 69,9 & 64,7 & - \\
\hline $\begin{array}{l}\text { Matières azotées totales } \\
(\mathrm{N} \times 6,25) \ldots \ldots \ldots \ldots \\
\text { Crude protein }\end{array}$ & 12,7 & 10,6 & 7,9 & 10,2 & 3,4 \\
\hline Matières minérales - Ash .... & 3,4 & 2,2 & 1,3 & 1,7 & 6,0 \\
\hline Calcium - Calcium ...... & 0,09 & 0,07 & 0,04 & 0,05 & 0,58 \\
\hline Phosphore - Phosphorus & 0,38 & 0,34 & 0,29 & 0,31 & 0,08 \\
\hline $\begin{array}{l}\text { Matière organique } \ldots \ldots \ldots \ldots \\
\text { Organic matter }\end{array}$ & 96,6 & 97,8 & 98,7 & 98,3 & 94,0 \\
\hline
\end{tabular}

Tablead $1 b$

Teneurs en composants pariétaux des céréales et de la paille (p. 100).

Fibre composition of cereals and straw (p. 100).

\begin{tabular}{|c|c|c|c|c|c|}
\hline & $\begin{array}{c}\text { Avoine } \\
\text { Oats }\end{array}$ & $\begin{array}{l}\text { Orge } \\
\text { Barley }\end{array}$ & $\begin{array}{l}\text { Maïs } \\
\text { Maize }\end{array}$ & $\begin{array}{c}\text { Blé } \\
\text { Wheat }\end{array}$ & $\begin{array}{l}\text { Paille } \\
\text { de blé } \\
\text { Wheat } \\
\text { straw }\end{array}$ \\
\hline $\begin{array}{l}\text { Fibres brutes (N.D.F.) ....... } \\
\text { Crude fibre }\end{array}$ & 33,6 & 11,2 & 11,1 & 7,9 & 76,6 \\
\hline Lignocellulose (A.D.F.) & 15,6 & 4,3 & 4,1 & 3,9 & 45,6 \\
\hline Hemicellulose (N.D.F.-A.D.F.) & 18,0 & 6,9 & 7,0 & 4,0 & 31,0 \\
\hline Lignine - Lignin $\ldots \ldots \ldots \ldots$ & 1,3 & 0,6 & 1,3 & 1,0 & 4,7 \\
\hline $\begin{array}{l}\text { Cellulose « vraie " } \\
\text { (A.D.F.-Lignine) ............ } \\
\text { "True » cellulose }\end{array}$ & 14,3 & 3,7 & 2,8 & 2,9 & 40,9 \\
\hline $\begin{array}{l}\text { Cellulose Weende } \ldots \ldots \ldots \ldots \\
\text { Crude cellulose }\end{array}$ & 12,9 & 4,2 & 2,0 & 2,6 & 40,7 \\
\hline
\end{tabular}




\section{TABleau $2 \mathrm{a}$}

Nature des régimes distribués (2000 g/animal/jour).

Crude composition of diets $(2000 \mathrm{~g} /$ animal/day).

\begin{tabular}{|c|c|c|c|c|}
\hline $\begin{array}{l}\text { Régime de base } \\
\text { Basal diet }\end{array}$ & $\begin{array}{l}\text { Avoine } \\
\text { Oats }\end{array}$ & $\begin{array}{l}\text { Orge } \\
\text { Barley }\end{array}$ & $\begin{array}{l}\text { Maïs } \\
\text { Maize }\end{array}$ & $\begin{array}{c}\text { Blé } \\
\text { Wheat }\end{array}$ \\
\hline $\begin{array}{l}\text { p. } 100 \mathrm{de} \\
\text { la consommation } \\
\text { p. } 100 \text { of feed intake }\end{array}$ & $\begin{array}{c}\text { Entière } \\
\text { Whole } \\
\text { Aplatie } \\
\text { Rolled }\end{array}$ & $\begin{array}{l}\text { Aplatie } \\
\text { Rolled } \\
\text { Trempée } \\
\text { Soaked }\end{array}$ & $\begin{array}{l}\text { Entier } \\
\text { Whole } \\
\text { Concassé } \\
\text { Crushed }\end{array}$ & $\begin{array}{l}\text { Entier } \\
\text { Whole } \\
\text { Aplati } \\
\text { Rolled }\end{array}$ \\
\hline Céréale - Cereal & 100 & 82,5 & 77,5 & 77,5 \\
\hline Paille de blé - Wheat straw ....... & 0 & 17,5 & 22,5 & 22,5 \\
\hline
\end{tabular}

TABleaU $2 b$

Composition analytique centésimale des régimes distribués. Analytical centesimal composition of diets.

\begin{tabular}{|c|c|c|c|c|}
\hline & $\begin{array}{l}\text { Avoine } \\
\text { Oat }\end{array}$ & $\begin{array}{l}\text { Orge } \\
\text { Barley }\end{array}$ & $\begin{array}{l}\text { Maïs } \\
\text { Maize }\end{array}$ & $\begin{array}{c}\text { Blé } \\
\text { Wheat }\end{array}$ \\
\hline Matière sèche - Dry matter & 89,3 & 92,1 & 89,1 & 89,0 \\
\hline \multicolumn{5}{|l|}{ p. 100 de M.S. } \\
\hline $\begin{array}{l}\text { Matières protéiques }(\mathrm{N} \times 6,25) \ldots . \\
\text { Crude protein }\end{array}$ & 12,7 & 9,4 & 6,9 & 8,7 \\
\hline Matière organique - Organic matter & 96,6 & 97,1 & 97,6 & 97,3 \\
\hline Amidon - Starch ... & 35,2 & 45,1 & 54,2 & 50,2 \\
\hline Calcium - Calcium ... & 0,09 & 0,16 & 0,16 & 0,16 \\
\hline Phosphore - Phosphorus ........ & 0,38 & 0,30 & 0,26 & 0,27 \\
\hline Fibres brutes (N.D.F.) - Crude fibre & 33,6 & 22,7 & 25,8 & 23,4 \\
\hline Lignocellulose (A.D.F.) & 15,6 & 11,5 & 13,5 & 13,3 \\
\hline Hémicelluloses (N.D.F.-A.D.F.) & 18,0 & 11,2 & 12,3 & 10,1 \\
\hline Lignine - Lignin .... & 1,3 & 1,3 & 2,1 & 1,8 \\
\hline $\begin{array}{l}\text { Cellulose "vraie» (A.D.F.-Lignine) } \\
\text { "True» cellulose }\end{array}$ & 14,3 & 10,2 & 11,4 & 11,5 \\
\hline $\begin{array}{l}\text { Cellulose Weende } \ldots \ldots \ldots \\
\text { Crude cellulose }\end{array}$ & 12,9 & 10,6 & 10,7 & 11,2 \\
\hline
\end{tabular}


Les matières premières, céréales et paille de blé, sont analysées par les mêmes méthodes. La composition analytique des régimes (tabl. 2 b) a été calculée à partir des résultats obtenus.

Parallèlement aux mesures de digestibilités, nous avons suivi la glycémie postprandiale, chez les poneys recevant l'avoine et l'orge, à partir de sang jugulaire prélevé avant le repas puis 2,4 et 6 heures après celui-ci. Le glucose est dosé par la glucose oxydase peroxydase, dans le sang comme sur le produit d'hydrolyse de l'amidon.

La lactacidémie a été déterminée pour les 4 régimes juste avant le repas puis 2 , 4 et 8 heures après celui-ci. L'acide lactique est dosé par voie enzymatique, à la lacticodéshydrogénase. variance.

Les résultats expérimentaux sont soumis au test de Student et à l'analyse de

\section{Résultats et discussion}

\section{A. Composition des aliments}

L'analyse des céréales et de la paille de blé (tabl. 1 a et 1 b) aboutit à des différences avec les valeurs généralement admises (I.N.R.A., 1978) pour les matières protéiques, les constituants cellulaires et les minéraux du blé et du maïs. La comparaison des 4 céréales étudiées (tabl. 1 a et 1 b) fait ressortir la richesse de l'avoine en constituants pariétaux, ce qui justifie l'adjonction de paille de blé dans les régimes comportant les autres céréales afin de les rendre comparables quant au taux de cellulose brute (tabl. 2 b). A ce propos, on peut noter, une fois de plus, la similitude entre les taux de cellulose Weende et de cellulose «vraie» (selon la méthode de Van Soest, 1963), quoique cette dernière soit un peu supérieure.

\section{B. Coefficients de digestibilités}

\section{Digestibilité des régimes}

L'étude des tableaux $3 \mathrm{a}$ et $3 \mathrm{~b}$ (moyennes et leurs écarts-types) conduit aux constatations suivantes :

- Le mode de présentation (entier, aplati, trempé ou concassé) n’a aucune influence sur la digestibilité des constituants d'une céréale donnée, excepté pour la cellulose vraie du régime à base de blé (l'aplatissage améliore alors cette digestibilité), et pour les matières minérales de façon plus générale.

Nous avons indiqué, à titre informatif, les digestibilités apparentes des minéraux et, en particulier du phosphore et du calcium. Elles sont sans grande signification, l'importance de la fraction endogène expliquant les valeurs négatives et la dispersion des résultats.

- La digestibilité apparente des protéines est tributaire du taux azoté. Ainsi, elle est plus élevée pour l'avoine et le régime à base d'orge que pour les régimes à base de maïs et de blé. 


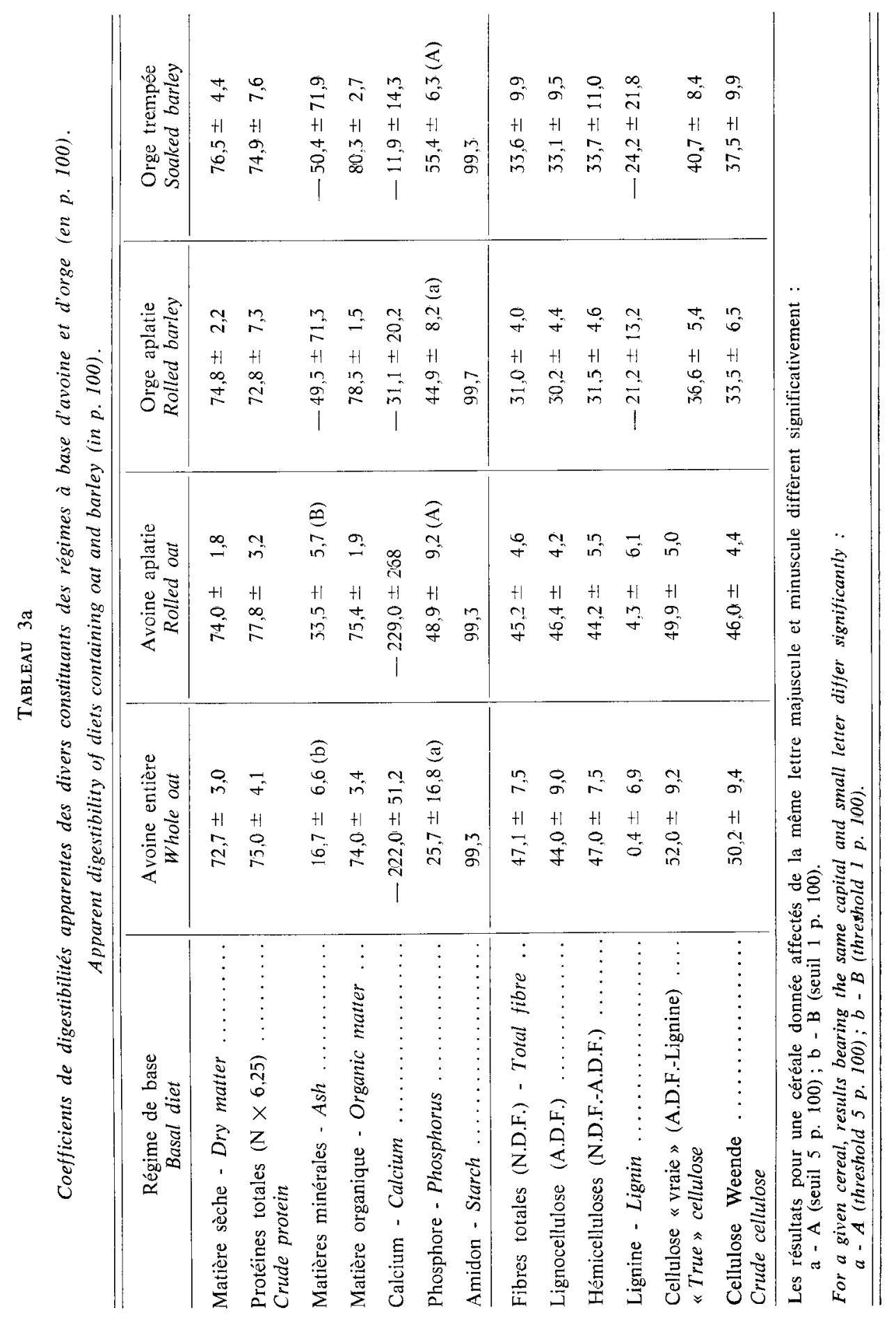




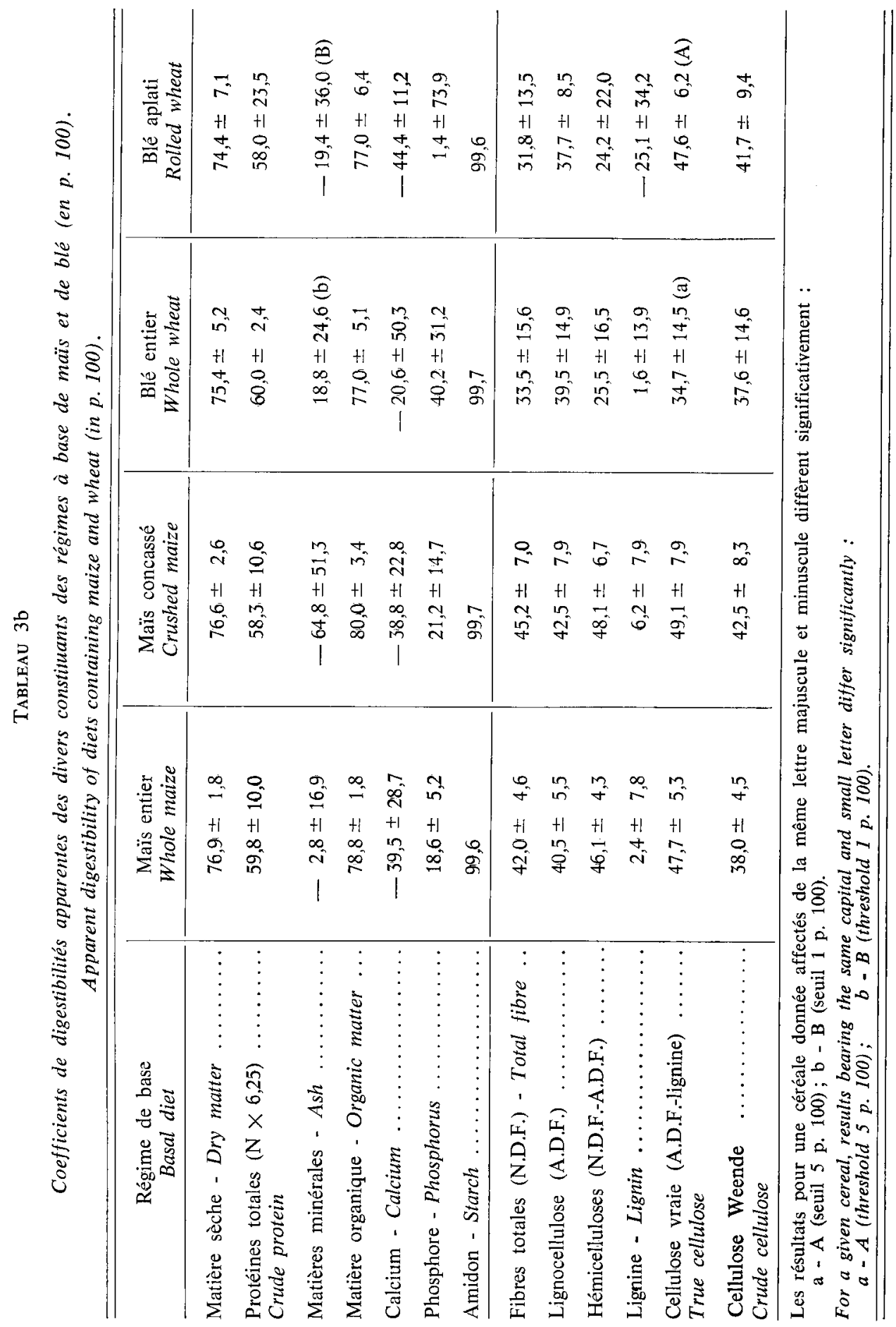


- Par contre, les digestibilités de la matière sèche et de la matière organique, qui traduisent sans doute le mieux la valeur alimentaire globale de l'aliment, ne diffèrent pas.

- La digestibilité des constituants pariétaux est relativement faible avec les régimes à base d'avoine ou de maïs, par rapport aux régimes à base d'orge ou de blé, ce qui n'est pas prévisible d'après la teneur des régimes en ces constituants.

- La digestibilité de la cellulose "vraie» (de Van Soest) est supérieure à celle de la cellulose brute (de Weende), de 2 à 5 points en moyenne, le régime à base de blé faisant exception. blé aplati.

- La lignine a une digestibilité proche de zéro, sauf pour l’orge et pour le

\section{Digestibilité des céréales}

Les différences - ou similitudes - observées quant aux digestibilités des régimes sont peut-être dues à la présence de paille de blé dont la digestibilité est très médiocre (Wolter et al., 1979). Aussi, connaissant la nature des régimes (tabl. 2 a), la composition analytique des céréales (tabl. 1 a et 1 b), et la digestibilité de la paille de blé, pouvons-nous calculer la digestibilité théorique relative à chaque céréale, selon la formule suivante :

$$
\mathrm{D} \text { céréale }=\frac{\mathrm{D} \text { régime } \times(\mathrm{E})_{\mathrm{r}}-\mathrm{D} \text { paille } \times(\mathrm{E})_{\mathrm{p}} \times(1-\mathrm{X})}{(\mathrm{E})_{\mathrm{C}} \times \mathrm{X}}
$$

avec :

- (E), quantité d'élément (exprimé en p. 100) contenu dans le régime $(R)$, la paille de blé (P) ou la céréale (C),

- $X$, pourcentage de céréale dans le régime, et $\mathrm{E}(\mathrm{R})=\mathrm{E}(\mathrm{C}) \times \mathrm{X}+\mathrm{E}(\mathrm{P}) \times(1-\mathrm{X})$.

Les coefficients de digestibilité des céréales, ainsi obtenus pour les 4 céréales étudiées, sont récapitulés dans le tableau 4. Ils négligent le phénomène de digestibilité associative qui peut biaiser les résultats, et restent hypothétiques. Aussi, nous nous limiterons à quelques remarques générales :

- Le mode de présentation n'a généralement pas d'influence sur la digestibilité apparente des céréales. Bouwman (1978) aboutissait à la même conclusion, en comparant l'avoine entière et l'avoine aplatie. CENNI et al. (1973) n'obtenaient qu'une très modeste amélioration de la digestibilité de la matière organique $(82,15$ p. 100 contre 80,70 p. 100) grâce au broyage de l'avoine.

Toutefois, l'aplatissage du blé accroît très sensiblement la digestibilité de sa cellulose brute (96 p. 100 au lieu de 73 p. 100), et surtout de sa cellulose «vraie» (99 p. 100 au lieu de 33 p. 100). Malgré tout, ces valeurs calculées par différence avec le régime comportant de la paille en complément a peu de signification pratique, ne serait-ce qu'en raison du faible taux de cellulose du blé.

- Les digestibilités respectives (tabl. 5) de la matière sèche (73,5 - 86 - 92 89,5 p. 100) et de la matière organique $(74,5-90-93,5-90,5$ p. 100) font ressortir, outre la correspondance entre ces deux paramètres, la supériorité des autres céréales vis-à-vis de l'avoine. 


\begin{tabular}{|c|c|c|c|c|c|c|c|c|c|c|c|c|c|c|}
\hline & 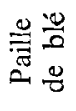 & 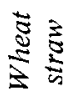 & $\stackrel{\infty}{i}$ & $=$ & $\begin{array}{l}\infty \\
\pm \\
\pm\end{array}$ & 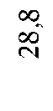 & $\begin{array}{c}\sigma \\
\dot{m}\end{array}$ & $\overrightarrow{1}$ & $\begin{array}{l}\infty \\
\infty \\
\sim\end{array}$ & $\overrightarrow{i^{\circ}}$ & $\frac{1 n}{m}$ & $\stackrel{m}{\infty}$ & $\vec{m}$ & $\begin{array}{l}\infty \\
\stackrel{\sim}{N}\end{array}$ \\
\hline & $\frac{\Xi}{\mathbb{Z}}$ & $\frac{\vec{\sigma}}{\frac{\sigma}{2}}$ & $\begin{array}{l}\stackrel{9}{0} \\
\infty \\
\infty\end{array}$ & $\overrightarrow{0}$ & $\begin{array}{c}+ \\
\text { in } \\
1\end{array}$ & $\ddot{g}$ & $\stackrel{\vec{T}}{T}$ & $\infty^{0}$ & m & $\stackrel{0}{i n}$ & $\stackrel{\infty}{n}$ & $\begin{array}{l}0 \\
\text { in } \\
1\end{array}$ & $\begin{array}{l}\infty \\
\infty \\
\infty\end{array}$ & 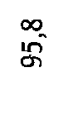 \\
\hline & $\dot{\ddot{\theta}}$ & 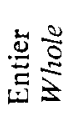 & ํ. & $\begin{array}{l}\sigma \\
8 \\
8\end{array}$ & $\hat{\tilde{N}}$ & "n & $\underset{1}{+্] ~}$ & $\ddot{m}$ & $\hat{o}$ & $\infty$ & $\stackrel{\circ}{\mathrm{i}}$ & $\stackrel{+}{m}$ & $\vec{m}$ & $\vec{N}$ \\
\hline$\dot{\widehat{s}}$ & 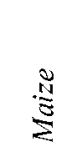 & 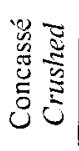 & $\hat{\vec{\sigma}}$ & $\frac{0}{6}$ & $\stackrel{n}{1}$ & 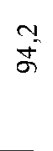 & i & 管 & $\vec{\infty}$ & $\bar{n}^{10}$ & $\frac{+}{8}$ & $\hat{i}$ & $\stackrel{\infty}{\stackrel{0}{0}}$ & $\stackrel{12}{E}$ \\
\hline 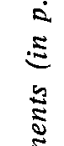 & 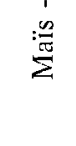 & 咅 & $\overline{\text { N }}$ & ro & $\begin{array}{c}\overrightarrow{0} \\
\dot{0}\end{array}$ & oi & 离 & $\stackrel{2}{\check{2}}$ & $\underset{8}{+}$ & $\vec{\infty}$ & $\begin{array}{l}\sigma \\
\text { वे }\end{array}$ & $\stackrel{\sigma}{\sigma}$ & $\vec{\rho}$ & $\begin{array}{l}1 \\
2 \\
\infty\end{array}$ \\
\hline $\begin{array}{l}\text { క్ } \\
8 \\
0 \\
\bar{\Xi}\end{array}$ & $\stackrel{\vec{\Xi}}{\stackrel{\Xi}{ \pm}}$ & 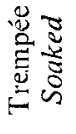 & $\underset{\infty}{0}$ & $\stackrel{8}{8}$ & $\begin{array}{c}\vec{\infty} \\
\infty \\
1\end{array}$ & $\begin{array}{l}\infty \\
\&\end{array}$ & $\stackrel{\text { Ś }}{1}$ & $\begin{array}{l}\text { ते } \\
\hat{b}\end{array}$ & $\begin{array}{l}0 \\
\text { P̊ }\end{array}$ & $\frac{12}{7}$ & $\begin{array}{l}\infty \\
10\end{array}$ & 总 & in $^{\infty}$ & $\mathrm{m}^{\mathrm{m}}$ \\
\hline 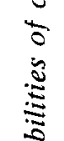 & $\stackrel{\infty}{\doteq}$ & 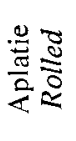 & $\begin{array}{l}0 \\
10 \\
\infty\end{array}$ & $\begin{array}{l}17 \\
\infty \\
\infty\end{array}$ & $\begin{array}{l}\hat{0} \\
0 \\
1\end{array}$ & $\begin{array}{l}0 \\
\infty \\
\infty\end{array}$ & $\frac{\text { 年 }}{1}$ & $\frac{\sigma}{i n}$ & 等 & $\vec{\infty}$ & $\frac{1 n}{m}$ & $\begin{array}{c}10 \\
\stackrel{n}{10} \\
1\end{array}$ & $\vec{\rho}$ & $\vec{F}$ \\
\hline 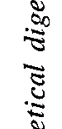 & $\stackrel{0}{\Xi}$ & $\begin{array}{l}\frac{\pi}{2} \\
\frac{\pi}{\pi} \\
\frac{\pi}{\pi}\end{array}$ & 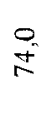 & $\stackrel{\infty}{\infty}$ & $i^{12}$ & $\stackrel{\vec{n}}{i}$ & $\begin{array}{c}\hat{a} \\
\vec{i}\end{array}$ & $\begin{array}{l}\sigma \\
\infty \\
\infty\end{array}$ & $\begin{array}{l}y \\
\text { wy }\end{array}$ & $\begin{array}{l}+ \\
\dot{g} \\
\dot{q}\end{array}$ & $\stackrel{\substack{7 \\
+}}{f}$ & $\stackrel{m}{*}$ & $\begin{array}{l}\sigma \\
\sigma\end{array}$ & 囱 \\
\hline 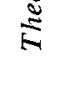 & 胥 & 总总 & $\hat{i}$ & $\stackrel{g}{g}$ & $\overbrace{0}^{\circ}$ & $\begin{array}{l}\tilde{z} \\
\tilde{i} \\
i\end{array}$ & $\stackrel{0}{ \pm}$ & $\hat{n}$ & 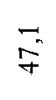 & $\stackrel{\circ}{f}$ & $\frac{8}{8}$ & $\stackrel{t}{0}$ & in & ్. \\
\hline & 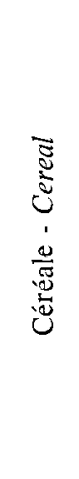 & 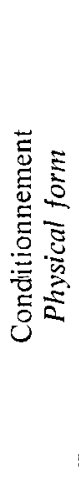 & 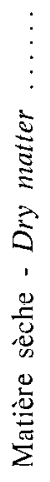 & 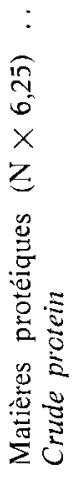 & 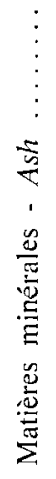 & 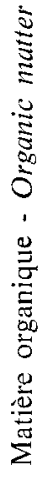 & 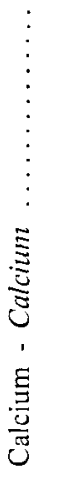 & 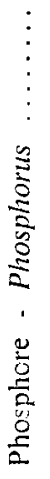 & 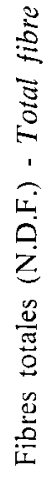 & 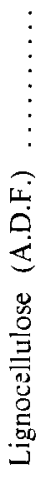 & 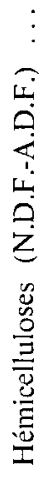 & 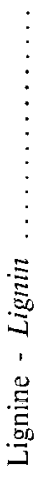 & 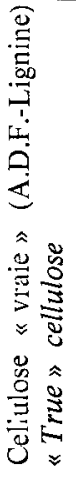 & 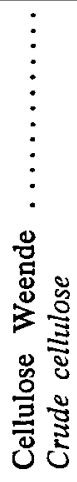 \\
\hline
\end{tabular}


R. WOLTER et al.

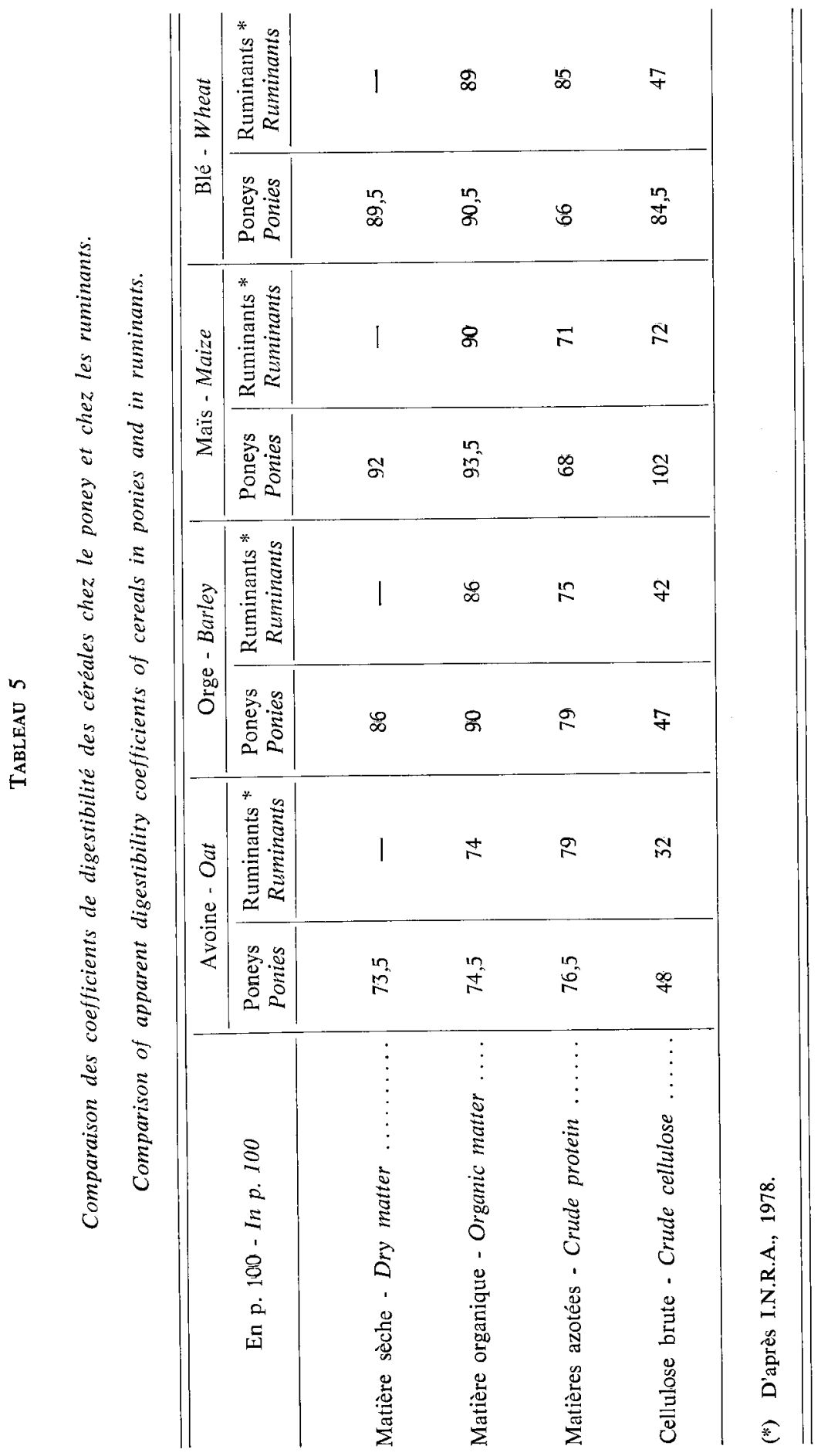


- Les céréales nues (maïs et blé), par rapport aux grains vêtus (orge et avoine), ont une plus médiocre digestibilité de l'azote (68 et 66 p. 100 au lieu de 76,5 et 79 p. 100) compte tenu des faibles teneurs des céréales utilisées mais une meilleure digestibilité de la cellulose brute (102 et 84,5 p. 100 contre 48 et 47 p. 100). (tabl. 5).

Ces résultats sont en bonne concordance avec ceux retenus pour les ruminants

\section{a) Evolution de la lactacidémie}

L'examen du tableau 6 nous indique que le taux d'acide lactique dans le sang parvient à son maximum entre 2 et 4 heures après les repas, à un niveau plus ou moins élevé selon les céréales, sans influence du mode de présentation.

\section{TABLEAU 6}

Evolution de la lactacidémie à la suite du repas de céréales.

Kinetics of blood lactic levels after the cereal meal.

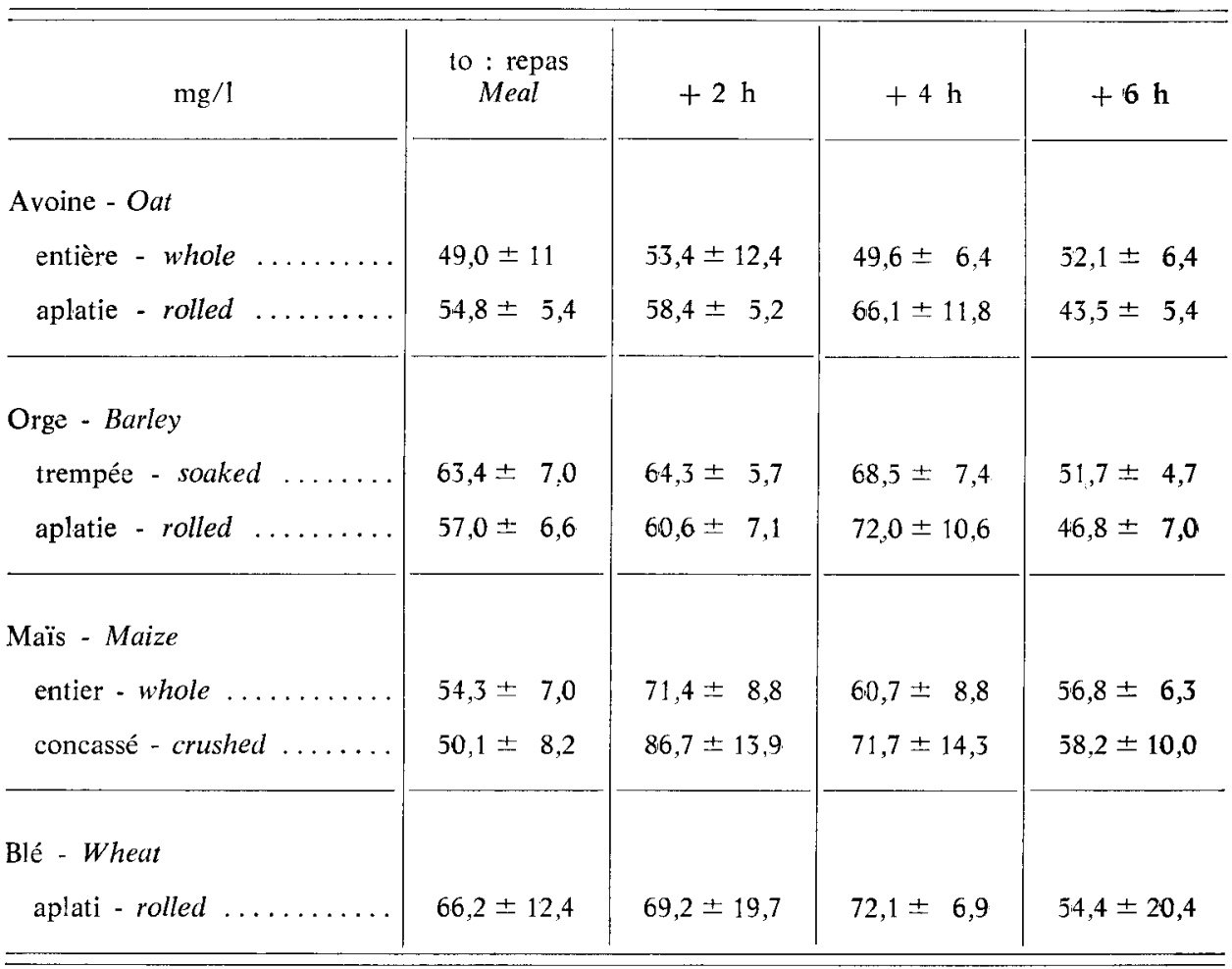


b) Evolution de la glycémie (tabl. 7)

La glycémic maximale qui est atteinte 2 heures après le repas, est de niveaux élevés et similaires avec les régimes avoine entière ou orge aplatie, mais plus faible lorsque les poneys consomment l'avoine aplatie ou l'orge trempée. Elle est aussi mieux maintenue avec l'avoine entière.

\section{TABLEAU 7}

Evolution de la glycémie postprandiale.

Kinetics of blood glucose levels after the meal.

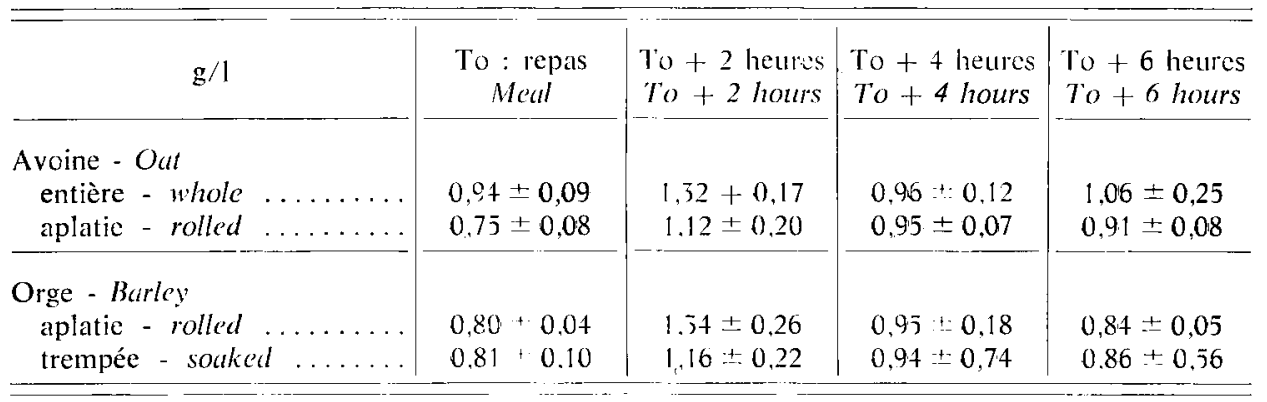

\section{Conclusion}

Dans les conditions de nos essais conduits chez des poneys, qui, par rapport au cheval, ont d'égales facultés digestives mais une consommation plus lente, et compte tenu d'un niveau d'ingestion relativement bas et fractionné en 2 repas journaliers, il apparaît que :

- Parmi les céréales étudiées : maïs, blé, orge, avoine, cette dernière se distingue par une plus faible digestibilité de ses constituants majeurs, et confirme ainsi sa moindre valeur énergétique.

- Le traitement des céréales par aplatissage, broyage ou trempage, semble généralement sans intérêt ; il ne peut donc avoir de justification que chez des équidés dont les tables dentaires sont altérées et la mastication insuffisante.

Accepté pour publication en juin 1982.

\section{Summary}

Compared digestibility of four cereals (oat, barley, maize and wheat) according to the physical form, in ponies

Six ponies, averaged $180 \mathrm{~kg}$, placed in metabolism crates, were fed alternately with cereals in different physical forms in four diets (oat alone or barley, maize and wheat with wheat straw) for total digestibilities $\left(40 \mathrm{~g} / \mathrm{kg} \mathrm{PV} \mathrm{PV}^{0.75} / \mathrm{day}\right.$ or $2000 \mathrm{~g} / \mathrm{animal} / \mathrm{day}$ in two meals). The analytical composition of cereals (Table 1a and $1 \mathrm{~b}$ ) was a little lower 
than usual. The composition of diets (Table $2 \mathrm{a}$ and $2 \mathrm{~b}$ ) tried to egalise the cell wall constituents.

Apparent digestibilities of diets (Table $3 a$ and $3 b$ ) showed no influence of the physical form, and those of cereals (Table 4 and 5) showed the lower energy value of oat.

Blood levels of lactic acid and glucose did not show difference between cereals (Table 6 and 7).

\section{Références bibliographiques}

Bouwman H., 1978. Digestibility trials with extruded feeds and rolled oats in ponies. Landbouwkund. Tijdschr., 90 (1), 2-6.

Cenni B., Jannella G.G., Colombani B., Verita P., 1973. Digestibility and feeding value of Maremma oats for ponies. Ann. Fac. Med. Vet. di Pisa, 26, 40-49.

Darlington J.M., Hershberger J.V., 1968. Effect de la maturité des fourrages sur la digestibilité, la consommation et la valeur nutritive de la luzerne, de la fléole et du dactyle. J. anim. Sci., 27, 1572-1576.

Fonnesbeck P.V., Lydman R.K., Vander Noot G.W., Symons I.D., 1967. Digestibility of the proximate nutrients of forage by horses. J. anim. Sci., 26, 1039-1045.

FonNesbeck P.V., 1968. Digestion of soluble and fibrous carbohydrate of forage by horses. J. anim. Sci., 27, 1336-1344.

FonNEsBeCK P.V., 1969. Partitioning the nutrients of forage for horses. J. anim. Sci., 28, 624-631.

Haenlein G.F.W., Holdren R.D., Yoon Y.M., 1966. Comparative response of horses and sheep to different physical forms of alfalfa hay. J. anim. Sci., 25, 740-743.

Hintz H.F., 1969. Comparaison des coefficients de digestibilité obtenus chez les bovins, les moutons, les lapins et les chevaux. The Veterinarian, 6 (1), 45-51.

HiNTz H.F., LoY R.G., 1966. Effects of pelleting on the nutritive value of horse ration. J. anim. Sci., 25, 1059-1063.

I.N.R.A., 1978. Alimentation des ruminants. Ed. I.N.R.A. Publications, route de SaintCyr, 78000 Versailles, 548-549.

Schurgi W.A., Frei D.L., Cheeke P.R., Holtan D.W., 1977. Utilization of whole corn pellets by horses and rabbits. J. anim. Sci., 45, 1317-1321.

Slade L.M., Hintz H.F., 1969. Comparaison de la digestion chez des chevaux, des poneys, des lapins et des cobayes. J. anim. Sci., 28, 842.

Vander Noot G.W., Gilbraith E.B., 1970. Digestibilité comparée des fourrages chez les chevaux et chez les bovins. J. anim. Sci., 31, 351-355.

Vander Noot G.W., Trout J.R., 1971. Prévision des composants digestibles des fourrages chez les équidés. J. anim. Sci., 33, 38-41.

VAN SoEst P.J., 1963. Use of detergent in the analysis of fibrous feed. II. - A rapid method for the determination of fiber and lignin. J. Assoc. off. anal. Chem., 46, 829-835.

Wolter R., Durix A., Letourneau J.C., 1974. Influence du mode de présentation du fourrage sur la vitesse du transit digestif chez le poney. Ann. Zootech., 23, 293-300.

Wolter R., Durix A., Letourneau J.C., 1975. Influence du mode de présentation du fourrage sur la digestibilité chez le poney. Ann. Zootech., 24, 237-242.

Wolter R., Chaabouni A., 1979. Etude de la digestion de l'amidon chez le cheval par analyse du contenu digestif après abattage. Rev. Méd. vét., 130 (10), 1345-1357.

Wolter R., Durix A., Letourneau J.C., Carcelen M., 1979. Evaluation chez le poney de la digestibilité du maïs-fourrage déshydraté, des pulpes sèches de betterave, de la luzerne déshydratée, du son de blé, de la paille de blé et des pulpes de raisins. Ann. Zootech., 28, 93-100.

Wolter R., Durix A., Letourneau J.C., Carcelen M., 1980. Evaluation chez le poney de la digestibilité des pellicules de soja, du marc de pommes, des caroubes et du tourteau de pépins de raisins. Ann. Zootech., 29, 377-385. 